\title{
La aplicación de las TIC en los procesos de enseñanza- aprendizaje en estudiantes de nivel medio superior en Tepic, Nayarit
}

\author{
The Application of ICT in the Teaching-Learning Processes by High School \\ Students in Tepic, Nayarit
}

\section{A aplicação das TIC nos processos de ensino-aprendizagem em estudantes do ensino médio de Tepic, Nayarit}

\author{
Gilberto Mejía Salazar \\ Universidad Autónoma de Nayarit, México \\ gilberto.mejia@uan.edu.mx \\ https://orcid.org/0000-0002-1879-1299
}

\section{Resumen}

La presente investigación tiene como objetivo principal difundir información estadística que permita conocer la utilidad de las herramientas tecnológicas aplicadas en el nivel medio superior en la ciudad de Tepic, Nayarit, México. Asimismo, analiza a través de los estudiantes el manejo y conocimiento de las tecnologías enfocadas en ambientes de aprendizaje y tecnológicos. Para determinar la muestra, se empleó la técnica de muestreo no probabilístico por conveniencia; para la recolección de la información, se aplicó un cuestionario estructurado con 10 preguntas cerradas a 150 estudiantes. La información obtenida fue procesada bajo el programa Statistical Package for the Social Sciences (SPSS) versión 19. Cabe mencionar que para el tratamiento y análisis de la información se crearon tablas de contingencia. Los resultados demuestran que la gran mayoría de los estudiantes utiliza constantemente la computadora para desarrollar tareas, trabajos de investigación; sobre todo, es un dispositivo electrónico de apoyo para los estudiantes y profesores dentro del plantel. Asimismo, los alumnos hacen uso de programas ofimáticos orientados a la 

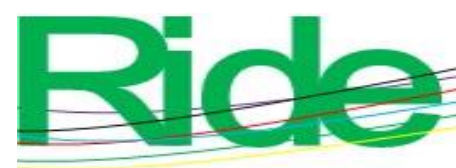

Revista Iberoamericana para la Investigación y el Desarrollo Educativo ISSN $2007-7467$

creación de documentos y presentaciones electrónicas. Se observa que este tipo de herramientas tecnológicas se adaptan a las necesidades educativas.

Palabras clave: ambientes de aprendizaje, bachillerato, educación de jóvenes, tecnología digital, tecnología educativa, TIC.

\section{Abstract}

The main objective of this research is to disseminate statistical information that allows knowing the usefulness of the technological tools applied at the upper middle level in the city of Tepic, Nayarit, México. Likewise, it analyzes through students the management and knowledge of technologies focused on learning and technological environments. To determine the sample, the non-probabilistic sampling technique was used for convenience; for the collection of information, a questionnaire structured by 10 closed-type questions was applied to 150 students. The information obtained was processed under the Statistical Package for the Social Sciences (SPSS) version 19 program. It is worth mentioning that for the treatment and analysis of the information contingency tables were created. The results show that the vast majority of students use constantly the computer to develop tasks, research papers; above all, it is an electronic support device for students and teachers on campus. Likewise, the students make use of office programs oriented to the creation of electronic documents and presentations. Finally, it is observed that this type of technological tools is adapted to the educational needs.

Keywords: learning environments, high school, youth education, digital technology, educational technology, ICT. 


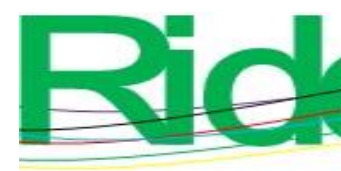

Revista Iberoamericana para la
Investigación y el Desarrollo Educativo ISSN 2007-7467

\section{Resumo}

O principal objetivo desta pesquisa é disseminar informações estatísticas que nos permitam conhecer a utilidade das ferramentas tecnológicas aplicadas no nível médio superior na cidade de Tepic, Nayarit, México. Da mesma forma, analisa através dos alunos a gestão e o conhecimento de tecnologias voltadas para ambientes de aprendizagem e tecnológicos. Para determinar a amostra, a técnica de amostragem não probabilística foi utilizada por conveniência; Para coletar as informações, foi aplicado um questionário estruturado com 10 perguntas fechadas a 150 alunos. As informações obtidas foram processadas sob o Statistical Package for the Social Sciences (SPSS) versão 19. É importante mencionar que foram criadas tabelas de contingência para o tratamento e a análise das informações. Os resultados mostram que a grande maioria dos estudantes utiliza constantemente o computador para realizar tarefas, trabalhos de pesquisa; Acima de tudo, é um dispositivo de suporte eletrônico para alunos e professores no campus. Da mesma forma, os alunos fazem uso de programas de automação de escritório orientados à criação de documentos e apresentações eletrônicas. Observa-se que esse tipo de ferramenta tecnológica se adapta às necessidades educacionais.

Palavras-chave: ambientes de aprendizagem, ensino médio, educação de jovens, tecnologia digital, tecnologia educacional, TIC.

Fecha Recepción: Febrero 2020

Fecha Aceptación: Julio 2020

\section{Introducción}

El universo de la educación ha cambiado profundamente: el nacimiento de una nueva era de enseñanza digital apuesta hacia la expresión humana social. De esta manera, la llegada de un nuevo paradigma de desarrollo social está entre nosotros. A ese desarrollo se le conoce como inclusión tecnológica (Petrella, 2001, citado en López, Damián, Garza y Rosales, 2017).

Colorado y Edel (2012) afirman que, en la actualidad, con los avances de la tecnología, el docente experimenta cambios en su estructura cognitiva en virtud de enfrentar nuevas formas de aprender para enseñar, es decir, tener acceso y aprender a utilizar los diferentes recursos tecnológicos como herramientas para el proceso de enseñanza-aprendizaje. 


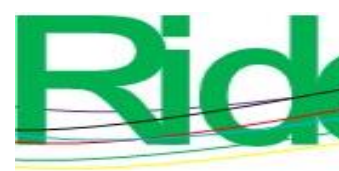
Revista Iberoamericana para la
Investigación y el Desarrollo Educativo
ISSN $2007-7467$

Y de acuerdo con Razo y Cabrero (2015):

Las tecnologías de información son un recurso cada vez más común en el trabajo de los estudiantes en clase, aunque son utilizadas principalmente como medios para la difusión y comunicación de datos, es decir, en proyecciones, en la exposición de la clase, en la explicación y resolución de preguntas y en actividades de transcripción (p. 26).

Teniendo en cuenta lo anterior, la presente investigación, realizada en la ciudad de Tepic, Nayarit, México, tiene como objetivo principal difundir información estadística que permita conocer la utilidad de las herramientas tecnológicas aplicadas en el nivel medio superior. Asimismo, analiza a través de los estudiantes el manejo y conocimiento de las tecnologías enfocadas en la enseñanza-aprendizaje.

Para cerrar este apartado diremos que, siguiendo a Hernandez (2017), los estudiantes actuales utilizan las herramientas tecnológicas para facilitar el aprendizaje, y que la búsqueda por mejorar el estudio trae consigo la tarea de involucrar la tecnología con la educación.

\section{Las TIC en la educación}

El uso de las tecnologías de la información y la comunicación (TIC) en el aula tiene un impacto que se manifiesta, según la visión de un grupo de profesores que participó en el trabajo de Muñoz (2011), en abrir la posibilidad a maestros y alumnos para acceder desde el aula a información remota, imágenes, videos, recursos auditivos, que facilitan los aprendizajes.

Asimismo, Zenteno y Mortera (2011) afirman que la integración de las TIC al proceso de enseñanza-aprendizaje implica cambios en la educación, en donde alumno y profesor necesitan incorporar habilidades y destrezas en el manejo de la tecnología educativa dentro del aula. De ahí que los docentes busquen información constantemente acerca de cómo utilizar las TIC en los procesos de enseñanza y aprendizaje (Kishi, Avril y Nonaka, 2014).

$\mathrm{Al}$ respecto, Morales (2013) define las competencias digitales que debe de tener un docente para el buen uso de las TIC dentro del aula, como se aprecia en la figura 1. 

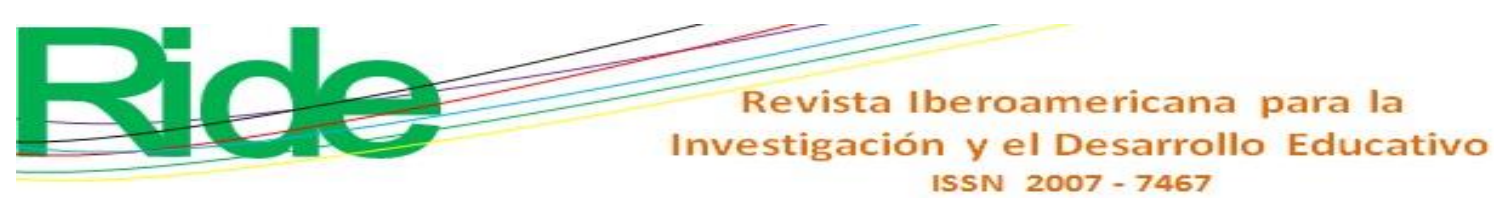

Figura 1. Competencias digitales del docente

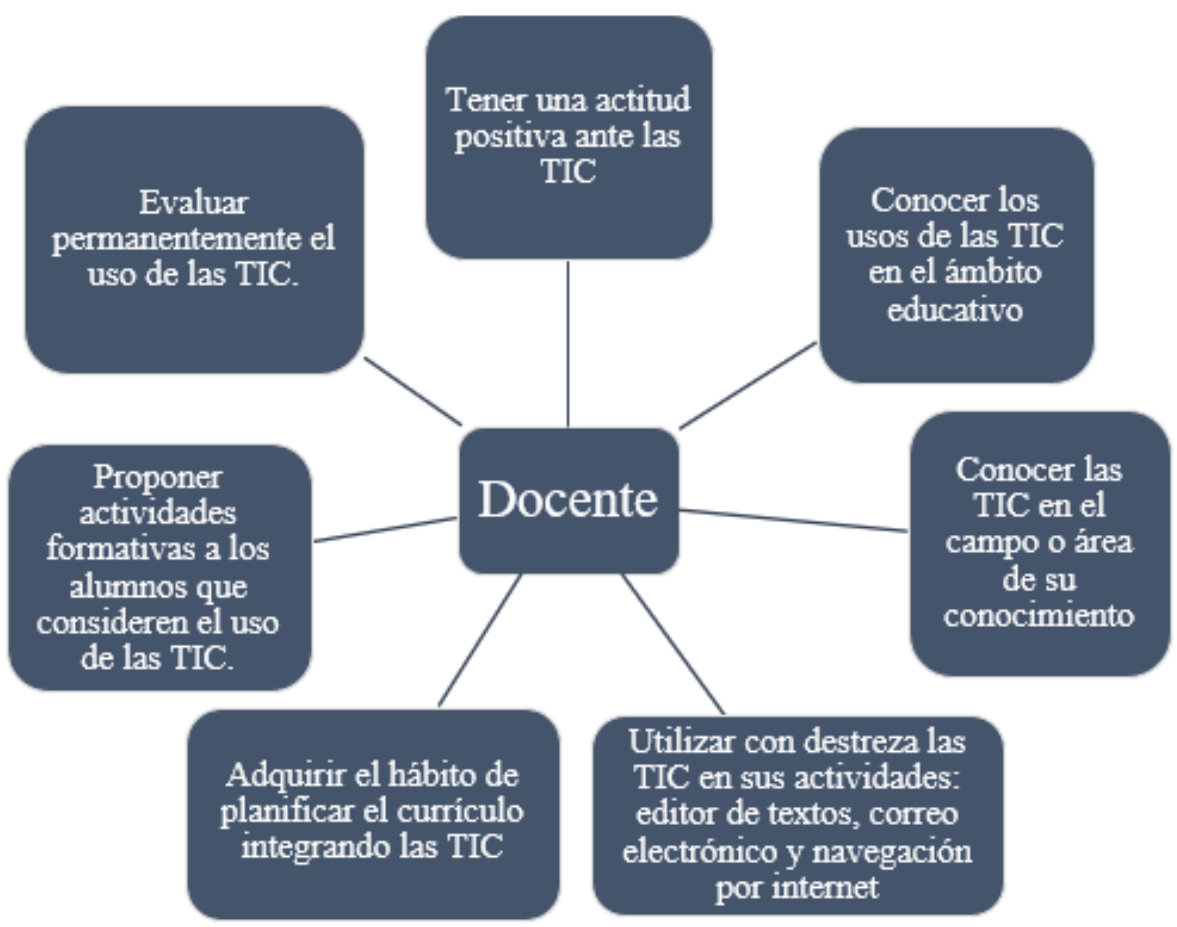

Fuente: Elaboración propia con base en Morales (2013)

Por lo tanto, las TIC son herramientas que han contribuido a la transformación de la sociedad en diversos aspectos, incluyendo, por supuesto, el de la educación, donde han tenido una fuerte incidencia. Un claro ejemplo son los alumnos que actualmente se están formando en las universidades e instituciones (Montoya, 2019). Cierto es que, de acuerdo con Kubota (2014), el uso de estas tecnologías en los sectores educativos varía, según la educación, sistemas, culturas escolares y principalmente los entornos tecnológicos.

\section{Las TIC como recurso de enseñanza-aprendizaje}

Con el fin de promover la aceptación de la tecnología, y mantener un entorno basado en las TIC, los docentes, los directores y coordinadores deberían de brindar apoyo técnico sobre el uso de estas herramientas, y de esta manera fomentar la educación (Terashima, Nakagawa, Kobayashi y Murai, 2019).

Asimismo, la capacitación encamina a las personas a tener un mejor desempeño en el manejo y conocimiento de estas herramientas electrónicas, entendiendo que esta, la capacitación, tiene por objetivo la mejora tanto en el proceso como en el producto final (Montoya, 2019). 


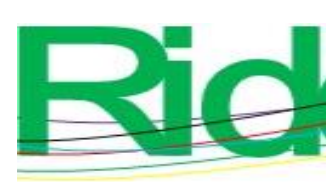
Revista Iberoamericana para la
Investigación y el Desarrollo Educativo
ISSN $2007-7467$

\section{Metodología}

Este trabajo se realizó con un enfoque descriptivo de tipo cuantitativo. Para determinar la muestra, se utilizó la técnica de muestreo no probabilístico por conveniencia, es decir, permite seleccionar aquellos casos accesibles que acepten ser incluidos (Otzen y Manterola, 2017). Para ilustrar mejor el muestreo por conveniencia, es aquel con el cual se seleccionan las unidades muestrales de acuerdo con la conveniencia o accesibilidad del investigador (Tamayo, 2001). Teniendo en consideración lo anterior, se determinó una muestra de 150 estudiantes.

\section{Método para el acopio de información}

Para la recolección de la información, se aplicó un cuestionario estructurado con 10 preguntas cerradas a estudiantes inscritos en el nivel medio superior, los cuales pertenecen a los grados de primero, segundo y tercer año de bachillerato, como se observa, a modo de ilustración, en la figura 2.

Figura 2. Alumnos respondiendo el cuestionario

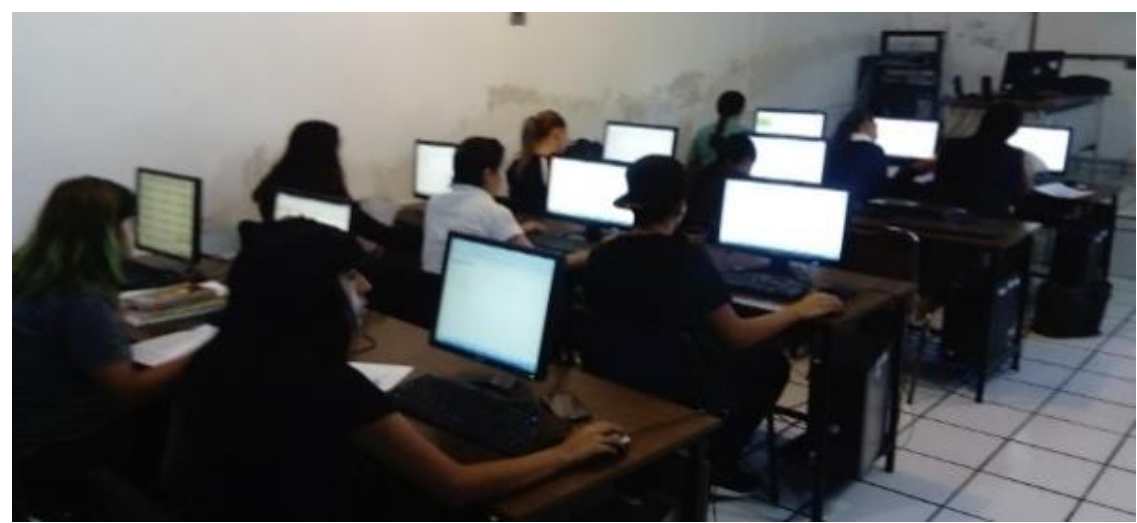

Fuente: Elaboración propia

La información obtenida fue procesada bajo el programa estadístico Statistical Package for the Social Sciences (SPSS) versión 19. Cabe mencionar que para el tratamiento y análisis de la información se crearon tablas de contingencia, en donde los resultados se presentan junto con sus respectivos gráficos.

La investigación se realizó en la ciudad de Tepic, ubicada en el estado de Nayarit, México. El estado colinda al norte con Sinaloa y Durango, y al este con Durango, Zacatecas y Jalisco (Aregional, 2009) (ver figura 3). 


\section{Revista Iberoamericana para la Investigación y el Desarrollo Educativo ISSN 2007 - 7467}

Figura 3. Ubicación geográfica de la ciudad de Tepic

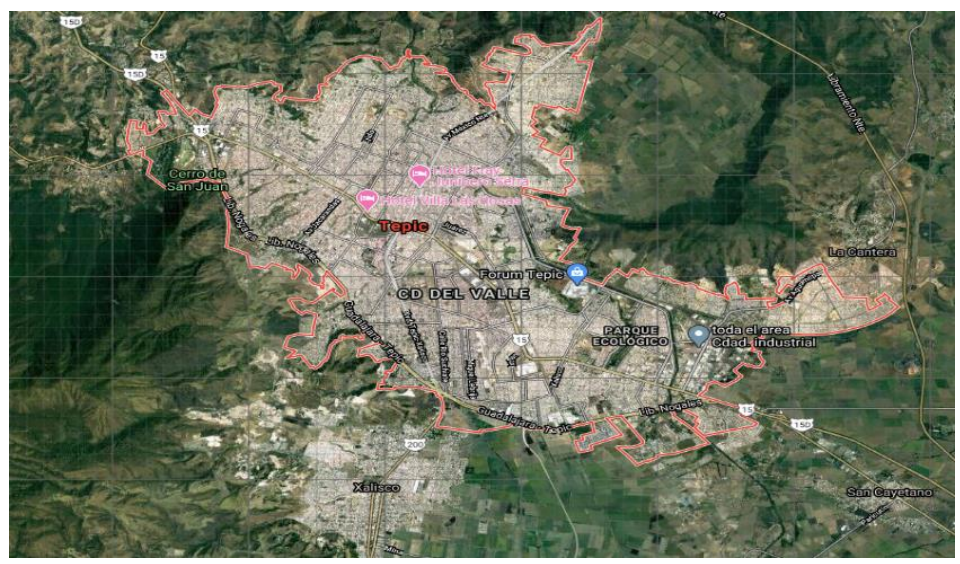

Fuente: Elaboración propia con base en imágenes del Google Maps (s. f.)

\section{Resultados}

A continuación, se presentan los resultados y la discusión del cuestionario aplicado.

De acuerdo con los resultados obtenidos, las edades de los alumnos de primer año son las siguientes: $24.7 \%$ tiene una edad de entre 15 y 25 años; $2.7 \%$ de entre 26 y 35 años, y $6 \%$ entre 36 y 45 años. En el segundo año, $24.7 \%$ tiene de 15 a 25 años; $6 \%$ de 26 a 35 años, y $2.7 \%$ de 36 a 45 años. En cuanto al tercer año, $17.3 \%$ se ubica en el rango 15-25 años; $8 \%$ en el de 26-35 años; $6 \%$ en el rango 36-45 años, y $2 \%$ en el rango de edad de 46-50 años. Estas cifras también se observan en la tabla 2.

Tabla 2. Edad de los estudiantes encuestados

\begin{tabular}{|c|c|c|c|c|}
\hline Edad & Primer año & Segundo año & Tercer año & Total \\
\hline 15 a 25 años & $24.7 \%$ & $24.7 \%$ & $17.3 \%$ & $66.7 \%$ \\
Recuento & 37 & 37 & 26 & 100 \\
\hline 26 a 35 años & $2.7 \%$ & $6 \%$ & $8 \%$ & $16.7 \%$ \\
Recuento & 4 & 9 & 12 & 25 \\
\hline 36 a 45 años & $6 \%$ & $2.7 \%$ & $6 \%$ & $14.7 \%$ \\
Recuento & 9 & 4 & 9 & 22 \\
\hline 46 a 50 años & $0 \%$ & $0 \%$ & $2 \%$ & $2 \%$ \\
Recuento & 0 & 0 & 3 & 3 \\
\hline
\end{tabular}

Fuente: Elaboración propia 

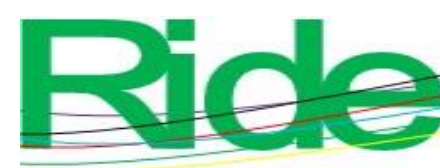

Revista Iberoamericana para la Investigación y el Desarrollo Educativo ISSN $2007-7467$

Con respecto al sexo, los resultados indican que, tomando en consideración el total de la muestra, los participantes de primer año del sexo masculino conforman la cifra de $10 \%$ y los de sexo femenino la de $23.3 \%$. En cuanto a los de segundo año, los de sexo masculino conforman la cifra de $13.3 \%$ y los del sexo femenino la de $20 \%$. Por último, del total, $10.7 \%$ son de sexo masculino y de tercer año y $22.7 \%$ de sexo femenino y de tercer año, tal y como se observa en la tabla 3 .

Tabla 3. Sexo de los estudiantes encuestados

\begin{tabular}{|c|c|c|c|c|}
\hline Sexo & Primer año & Segundo año & Tercer año & Total \\
\hline Masculino & $10 \%$ & $13.3 \%$ & $10.7 \%$ & $34 \%$ \\
Recuento & 15 & 20 & 16 & 51 \\
\hline Femenino & $23.3 \%$ & $20 \%$ & $22.7 \%$ & $66 \%$ \\
Recuento & 35 & 30 & 34 & 99 \\
\hline
\end{tabular}

Fuente: Elaboración propia

\section{Uso de las tecnologías}

Ahora bien, en cuanto a la pregunta "¿Con qué frecuencia utilizas un equipo de cómputo, o tu propio equipo, durante tus estudios?", los resultados para primer año fueron: $12.7 \%$ todos los días, $6 \%$ una vez por semana, $10.7 \%$ dos a tres veces a la semana, $0.7 \%$ mensualmente y $3.3 \%$ rara vez. Para segundo año, $10.7 \%$ indicó que todos los días, $6 \%$ una vez por semana, $13.3 \%$ de dos a tres veces a la semana y $3.3 \%$ mencionó que rara vez. Para tercer año, $10.7 \%$ respondió que todos los días, $6.7 \%$ una vez por semana, $14.7 \%$ dos a tres veces a la semana y $1.3 \%$ rara vez utiliza un equipo de cómputo, como se observa en la figura 4. 

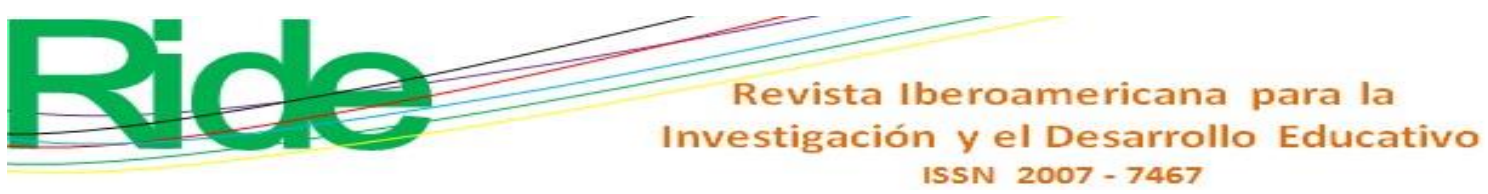

Figura 4. ¿Con qué frecuencia utilizas un equipo de cómputo, o tu propio equipo, durante tus estudios?

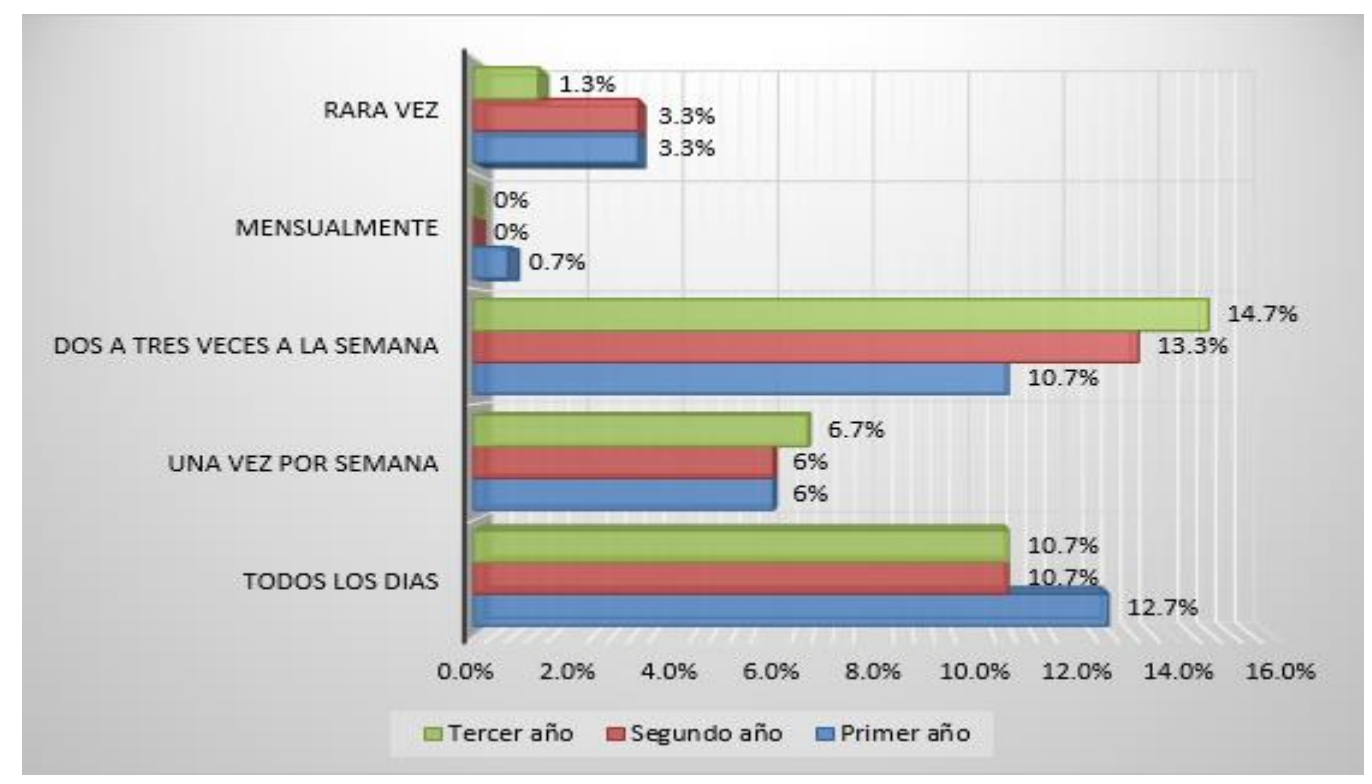

Fuente: Elaboración propia

Al cuestionamiento "¿Con qué frecuencia el profesor te pide que hagas uso de las tecnologías (Internet, motores de búsqueda, programas, tutoriales, email, foros, etc.)?, $0.7 \%$ de los participantes de primer año mencionó que nunca, $13.3 \%$ respondió que ocasionalmente, $14.7 \%$ que constantemente y $4.7 \%$ de manera muy constante frecuenta este tipo de tecnologías. Respecto a los de segundo año, $0.7 \%$ de los participantes se decantó por decir que nunca, $13.3 \%$ mencionó que ocasionalmente, $12.7 \%$ constantemente y $6.7 \%$ muy constantemente. Por último, los estudiantes de tercer año en $5.3 \%$ mencionaron que ocasionalmente, $19.3 \%$ dijo que constantemente y $8.7 \%$ contestó que de modo muy constante, tal y como se observa en la figura 5. 

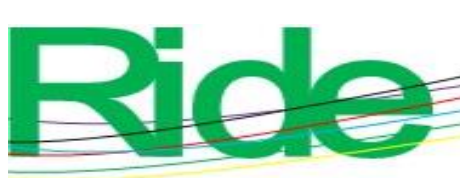

Revista Iberoamericana para la Investigación y el Desarrollo Educativo ISSN $2007-7467$

Figura 6. ¿Utiliza dispositivos tales como proyector, computadoras para realizar actividades dentro del aula?

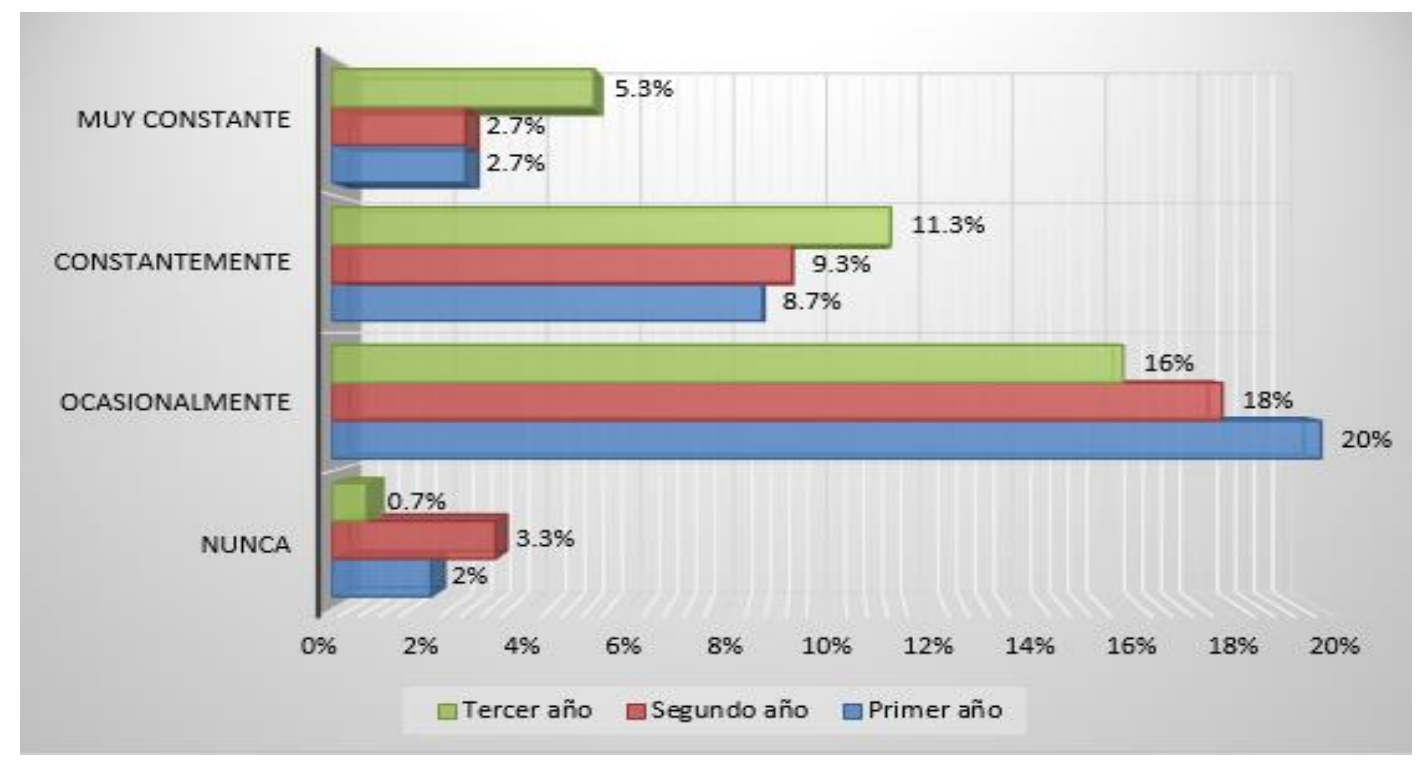

Fuente: Elaboración propia

Respecto al ítem “¿Hace uso de la computadora para desarrollar tareas en equipo?”, de los estudiantes encuestados de primer año, $2 \%$ respondió que nunca, $12 \%$ ocasionalmente, $13.3 \%$ constantemente y $6 \%$ mencionó que de manera muy constante.

Por su parte, $2.7 \%$ de los alumnos de segundo año dijo que nunca, $16.7 \%$ que ocasionalmente, $11.3 \%$ expresó que constantemente y $2.7 \%$ dijo que muy constantemente. Por último, $4 \%$ de los participantes de tercer año dijo que ocasionalmente, $18.7 \%$ se decantó por responder que constantemente y $10.7 \%$ confesó que lo hace de manera muy constante.

Cabe mencionar que la gran mayoría de los estudiantes utiliza constantemente la computadora para desarrollar tareas, trabajos e investigación; sobre todo, es un dispositivo electrónico de apoyo para los estudiantes y profesores dentro del plantel, como se aprecia en la figura 7 . 


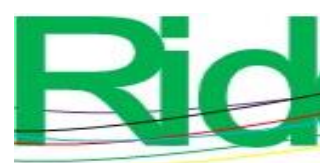

\section{Revista Iberoamericana para la Investigación y el Desarrollo Educativo ISSN $2007-7467$}

Figura 7. ¿Hace uso de la computadora para desarrollar tareas en equipo?

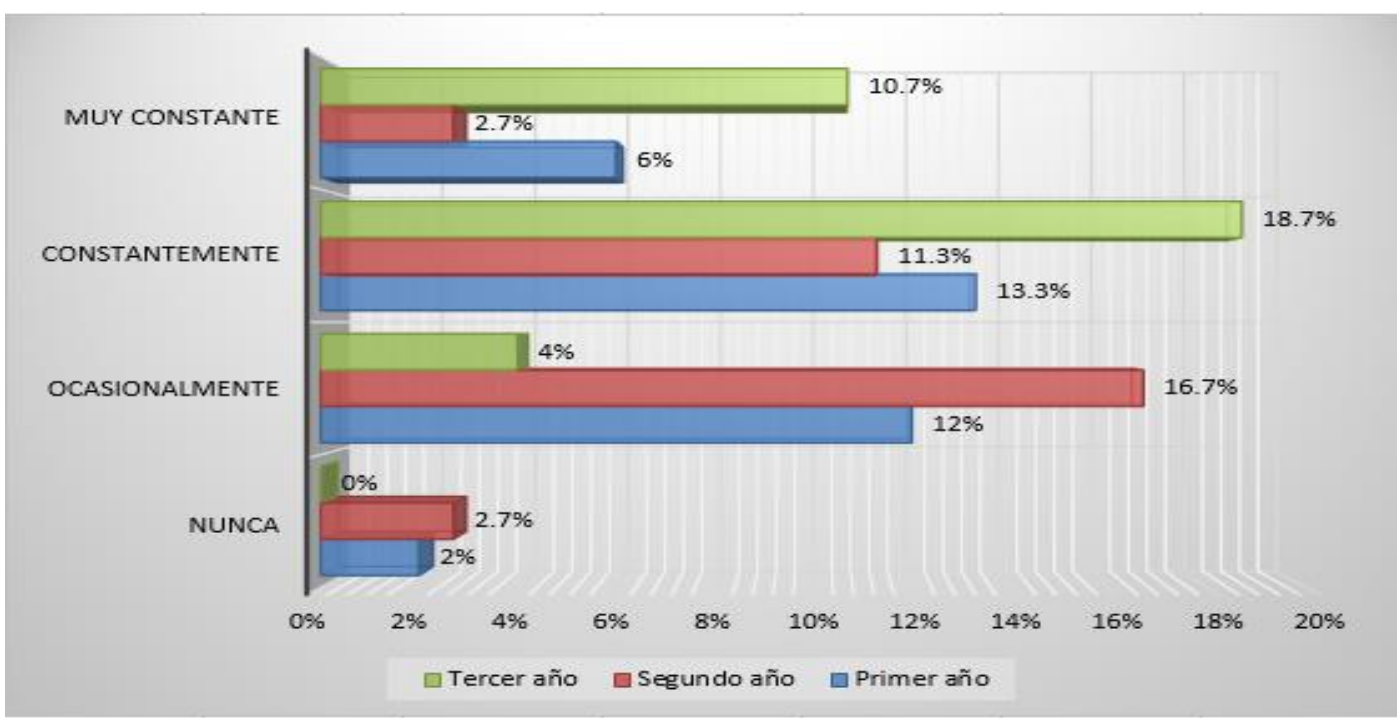

Fuente: Elaboración propia

Los resultados a la pregunta “¿Ha enviado tareas o cualquier otro tipo de documentación adjunta a través del correo electrónico (email)?" son los siguientes: $11.3 \%$ de primer año dijo que nunca ha enviado documentación adjunta, $16.7 \%$ que ocasionalmente y $5.3 \%$ que constantemente. En cuanto al conjunto de segundo año, $6 \%$ dijo que nunca, $20 \%$ ocasionalmente, $5.3 \%$ constantemente y $2 \%$ muy constantemente. De tercer año, $1.3 \%$ mencionó que nunca, $12.7 \%$ que ocasionalmente, $15.3 \%$ constantemente y $4 \%$ muy constantemente (véase figura 8 ).

Figura 8. ¿Ha enviado tareas o cualquier otro tipo de documentación adjunta a través del correo electrónico (email)?

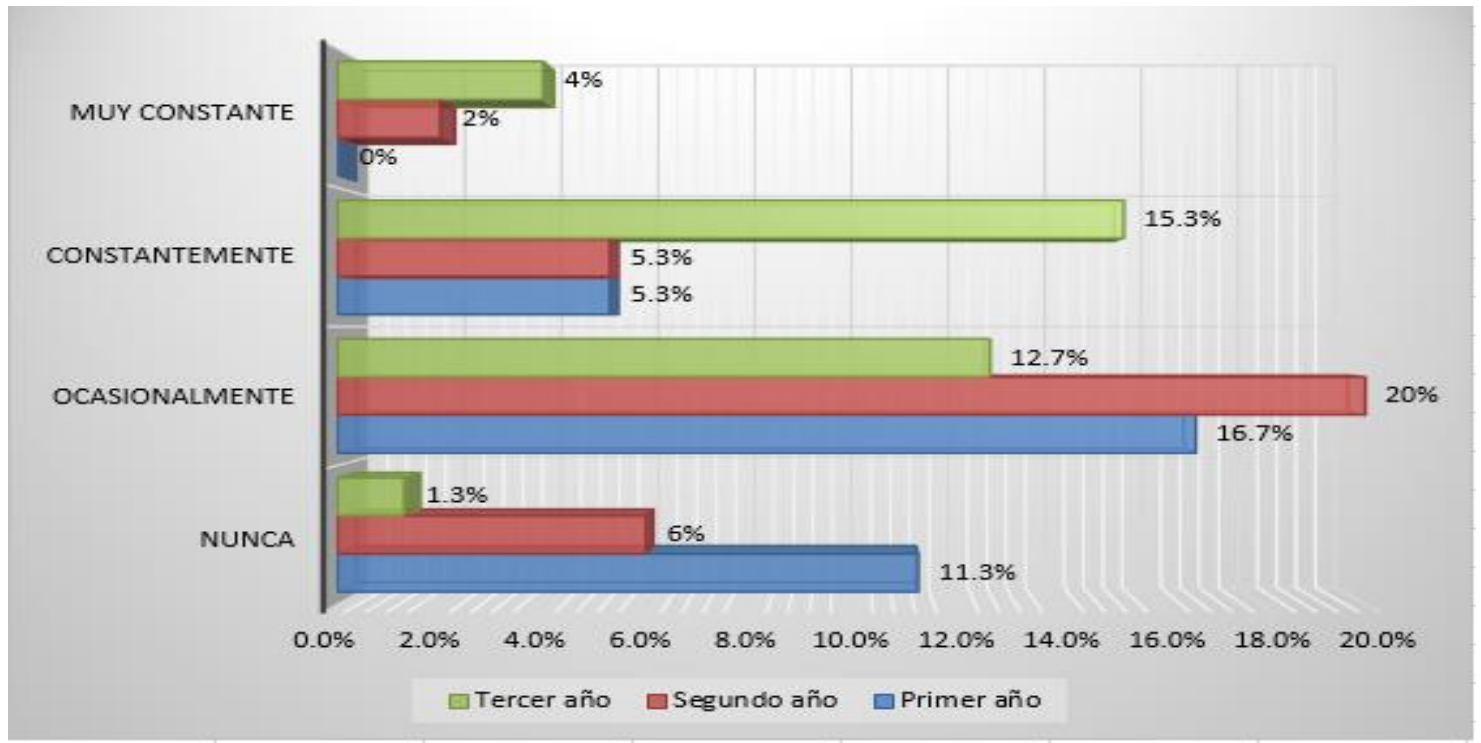

Fuente: Elaboración propia 


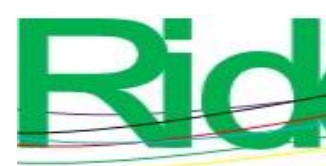

\section{Revista Iberoamericana para la Investigación y el Desarrollo Educativo ISSN $2007-7467$}

“Ha descargado recursos electrónicos disponibles en Internet para desarrollar sus actividades académicas?" fue la pregunta que continuó, y a la que $6 \%$ de los estudiantes de primer año $6 \%$ respondió que nunca ha descargado recursos de la Red, $9.3 \%$ ocasionalmente, $14 \%$ constantemente y $4 \%$ muy constante. Respecto a los de segundo año, $5.3 \%$ dijo que nunca, $14 \%$ que ocasionalmente, $7.3 \%$ expresó que hace esto constantemente y $6.7 \%$ muy constantemente. Finalmente, $0.7 \%$ de los alumnos de tercer año expresó que nunca, $12 \%$ ocasionalmente, $15.3 \%$ constantemente y $5.3 \%$ muy constantemente.

Los resultados demuestran que los estudiantes hacen uso de estos recursos disponibles y que gracias a ello tienen un mejor desempeño dentro de la escuela, como se aprecia en la figura 9.

Figura 9. ¿Ha descargado recursos electrónicos disponibles en Internet para desarrollar sus actividades académicas?

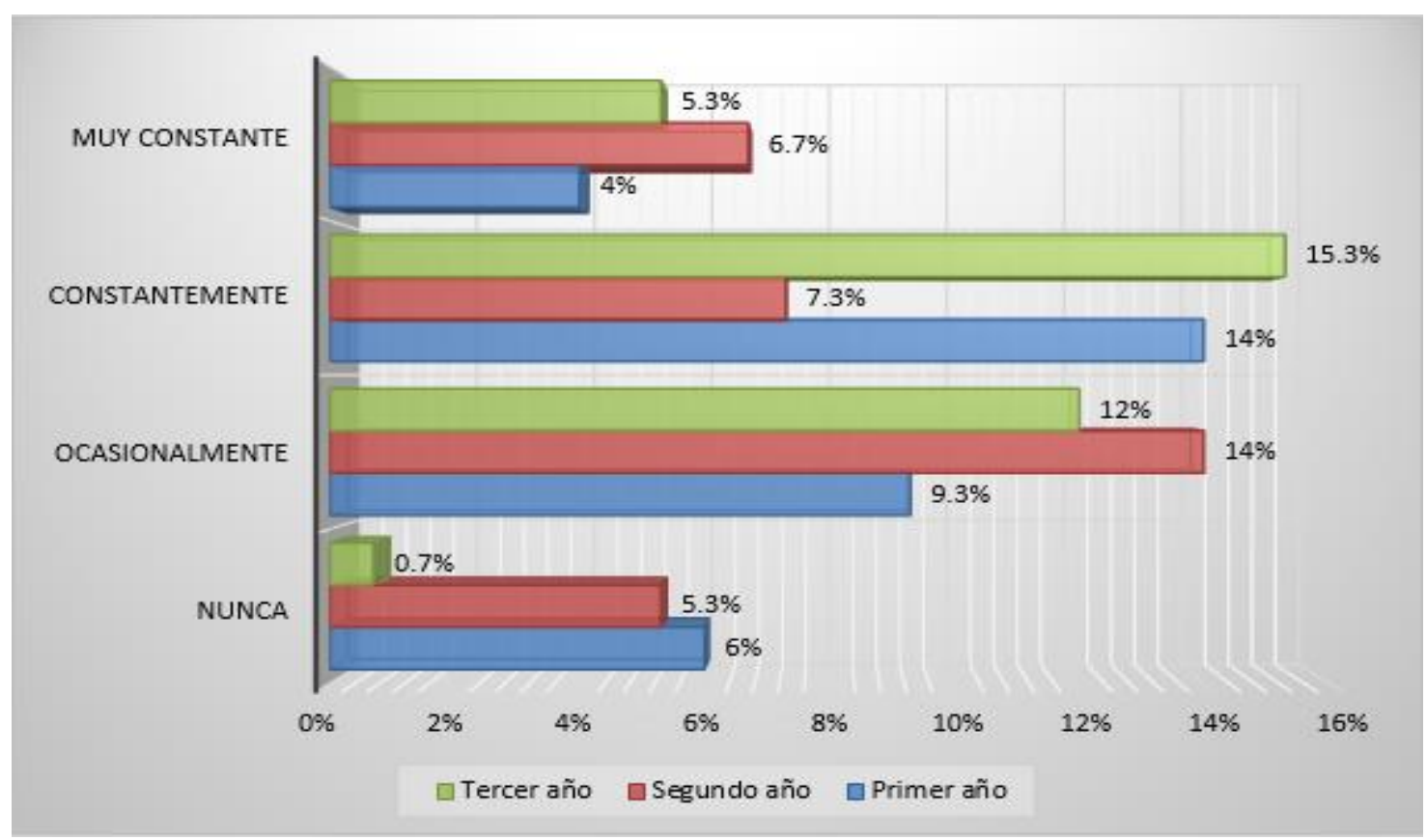

Fuente: Elaboración propia

Ahora bien, $7.3 \%$ de los estudiantes de primer año respondió que los profesores nunca han proporcionado materiales de uso electrónico para impartir la clase, $15.3 \%$ dijo que ocasionalmente, $8.7 \%$ constantemente y $2 \%$ de manera muy constante. Respecto a los de segundo año, $2 \%$ dijo que nunca, $14.7 \%$ ocasionalmente, $12.7 \%$ constantemente y $4 \%$ que lo hacen de modo muy constante. Los estudiantes de tercer año refirieron que $1.3 \%$ los profesores no proporcionan materiales de uso electrónico, $15.3 \%$ que lo hacen ocasionalmente, $12.7 \%$ constantemente y $4 \%$ muy constante, como se aprecia en la figura 10. 

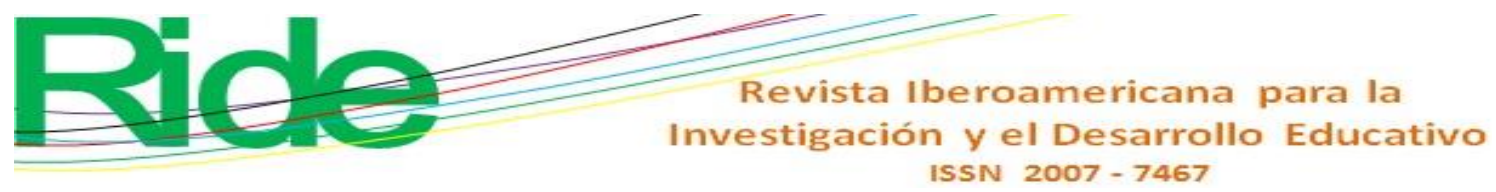

Figura 10. ¿Los profesores han proporcionado materiales de uso electrónico para impartir la clase?

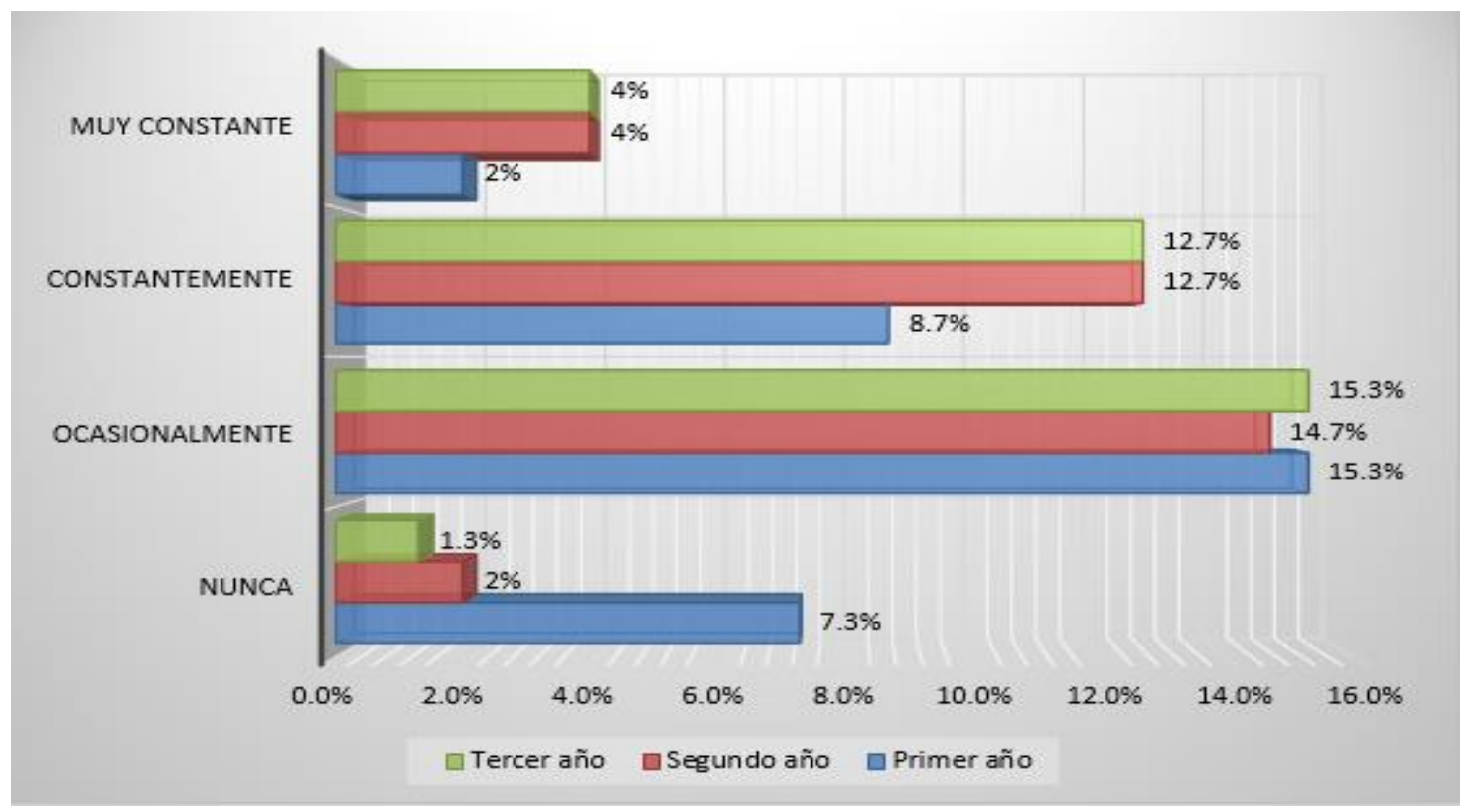

Fuente: Elaboración propia

Finalmente, los resultados demuestran que los estudiantes de los diferentes grados hacen uso de programas orientados para la creación de documentos y presentaciones electrónicas. Esto se entiende ya que este tipo de programas ofimáticos se adaptan a las necesidades de los escolares, por lo que son de gran apoyo en su formación académica, como se observa en la figura 11.

Figura 11. ¿Cuál de estos programas utiliza constantemente para sus actividades académicas?

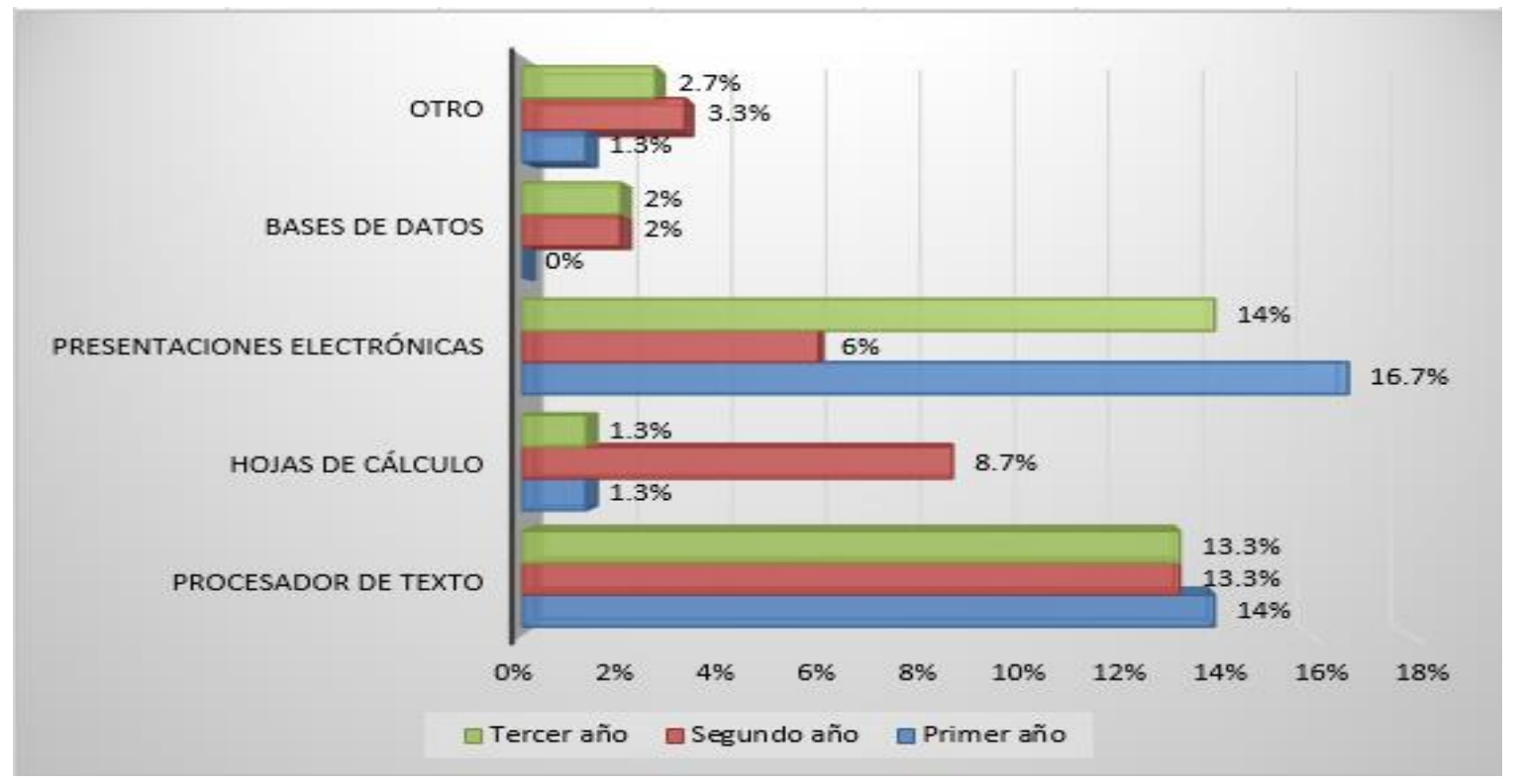

Fuente: Elaboración propia 


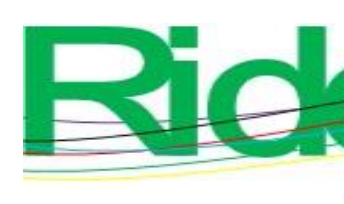
Revista Iberoamericana para la
Investigación y el Desarrollo Educativo
ISSN $2007-7467$

\section{Discusión}

Los siguientes resultados demuestran que la edad promedio de los alumnos involucrados que utilizan este tipo de herramientas tecnologías oscila entre los 15 y 25 años, rango en el que entra $66.7 \%$ del total de la población encuestada. Asimismo, que $66 \%$ los estudiantes del sexo femenino están más familiarizados con este tipo de herramientas. Vale la pena señalar que el uso constante de estas tecnologías abre una brecha digital para el desempeño y desarrollo de las actividades escolares y sociales que se demandan hoy en día en la esfera educativa.

Así pues, con los 150 estudiantes encuestados, se determinó que para los tres grados de bachillerato la frecuencia con que utilizan un equipo de cómputo durante sus estudios es de dos a tres veces a la semana (38.7\%). Además, $46.7 \%$ es constante con el uso de las tecnologías, por ejemplo, el manejo de motores de búsqueda, programas, videotutoriales, email, herramientas ofimáticas, etc.

El área más débil que se observó fue el uso de los dispositivos tales como el proyector o la propia computadora dentro del aula. En este sentido, el profesorado debe alentar el uso de los dispositivos electrónicos dentro del aula de clase, por medio de actividades concurrentes con este tipo de tecnologías, o implementando talleres para fortalecer la destreza con estas.

También se preguntó al estudiante si ha enviado documentación adjunta por medio del correo electrónico, y se encontró que 49.4 \% hace un uso ocasional con este tipo de herramientas.

Una de las fortalezas encontradas en esta investigación fue el manejo de los recursos disponibles dentro en la Red por parte de los participantes: se aprecia que $36.6 \%$ de los estudiantes descarga este tipo de recursos para la complementación de tareas y trabajos de investigación, entre otros.

Finalmente, los alumnos de los diferentes grados hacen uso de programas ofimáticos para la creación de documentos y presentaciones electrónicas, debido a que este tipo de programas se adapta a las necesidades de los escolares, así como a la implementación de trabajo colaborativo entre ellos. 


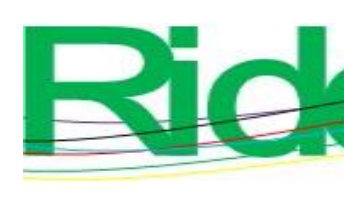

Revista Iberoamericana para la Investigación y el Desarrollo Educativo ISSN $2007-7467$

\section{Conclusiones}

Ciertamente, vivimos en un mundo donde las nuevas tecnologías están revolucionando la forma de enseñar y aprender. Las TIC han tomado el papel de herramientas de apoyo para la docencia. Son de gran utilidad en las instituciones educativas privadas y públicas, sin dejar de lado la parte social, donde juegan un papel importante. Ahora resulta difícil concebir la educación sin la inclusión de estas herramientas.

Los resultados encontrados en esta investigación demuestran que los estudiantes están familiarizados con este tipo de tecnologías, principalmente para la gestión y manipulación de la información. Se observa que, del total de estudiantes encuestados, $98.7 \%$ hace uso de ellas; muestran tener capacidad en el manejo de Internet, motores de búsqueda, programas, tutoriales, email, foros, etc. De esta manera, se conoce que los estudiantes recurren a los recursos electrónicos disponibles dentro de la Red necesarios para su desarrollo académico.

En esa línea, $95.3 \%$ de los estudiantes encuestados utiliza la computadora como una herramienta tecnológica primaria para el desarrollo de tareas o trabajos de investigación, y sobre todo es un dispositivo de apoyo para los estudiantes y profesores dentro del plantel; sin embargo, no hay que perder de vista que el porcentaje restante, $4.7 \%$, no hace uso de estas tecnologías para las actividades escolares o simplemente le resta importancia al beneficio que otorgan este tipo de herramientas.

Asimismo, los estudiantes hacen uso de programas ofimáticos orientados a la resolución de actividades básicas y comunes. Al respecto, $40.6 \%$ de los estudiantes emplea procesadores de texto para la creación de documentos y otro tipo de documentación necesaria, mientras que $36.7 \%$ requiere trabajar con presentaciones electrónicas para la exposición de trabajos de investigación y trabajo en equipo dentro del aula de clase; no menos importante es el dato de que $22.6 \%$ refiere hacer uso de las hojas de cálculo para tareas enfocadas a la estadística, base de datos para la gestión y manipulación de la información.

En suma, atendiendo a las preguntas de investigación planteadas con anterioridad, gran parte de la educación está enfocada en el uso y conocimiento de las TIC, a partir de lo cual es posible tener estudiantes autodidactas capacitados para la resolución de problemas y gestión de la información, y conformar grupos de estudio y alcanzar la colaboración que requiere el trabajo en equipo, todo lo cual abre una brecha hacia una educación tecnológica y continua dentro del plantel. 


\section{Referencias}

Aoki, K. (2010). The Challenges of ICT applications in distance Higher Education in Japan. Asian Journal of Distance Education, 8(2), 29-39.

Aregional. (2009). Estado de Nayarit (Monografía). Aregional. Recuperado de http://www.aregional.com/doc/pdf/nayarit.pdf.

Colorado, B. L. y Edel, R. (2012). La usabilidad de TIC en la práctica educativa. RED. Revista de Educación a Distancia, (30), 1-11.

Garza, E. (2013). Uso y consumo de Internet en jóvenes estudiantes: análisis del estado de Tamaulipas. (tesis doctoral). Universidad de Santiago de Compostela, España.

Google Maps (s. f.). Mapa de Tepic, Nayarit, México. Google Maps. Recuperado de https://www.google.com.mx/maps/place/Tepic,+Nay./@21.501062,-104.9119242,13941m/data=!3m2!1e3!4b1!4m5!3m4!1s0x842735d8893dcfdb:0x8378464 $5 f 40 \mathrm{c} 2 \mathrm{~d} 79 ! 8 \mathrm{~m} 2 ! 3 \mathrm{~d} 21.5041651 ! 4 \mathrm{~d}-104.8945887$

Hernandez, R. M. (2017). Impacto de las TIC en la educación: Retos y Perspectivas. Propósitos y Representaciones, $\quad 5(1), \quad 325-347 . \quad$ Recuperado de http://dx.doi.org/10.20511/pyr2017.v5n1.149.

Instituto Nacional de Estadística y Geografía [Inegi]. (2015). Estadísticas a propósito del día mundial de internet (17 de mayo). Datos de Nayarit. México: Instituto Nacional de $\begin{array}{lllll}\text { Estadística } & \text { y } & \text { Geografía. } & \text { Recuperado de }\end{array}$ https://www.inegi.org.mx/contenidos/saladeprensa/aproposito/2015/internet18.pdf.

Kishi, M., Avril, L. and Nonaka, Y. (2014). Socio-Cultural features of ICT practice at primary schools: from teachers' perceptions about UK and Japanese ICT use. International Journal for Educational Media and Technology, 8(1), 11-23.

Kubota, M. (2014). The passive usage of ICT by Japanese undergraduate students. International Journal for Educational Media and Technology, 8(1), 41-55.

López, B., Damián, J., Garza, F. y Rosales, J. (2017). Los estudiantes de Educación Media Superior y las TIC: situación de estudiantes oaxaqueños de 21 instituciones. Oaxaca, México: Universidad del Papaloapan. Recuperado de https://www.unpa.edu.mx/libros/3estudiantesTIC.pdf.

Montoya, L. M. (2019). La incorporación de las TIC en la capacitación docente. Estudio de caso: Universidad Autónoma Chapingo. Revista Electrónica sobre Ciencia, Tecnología y Sociedad (CTES), 6(11). 


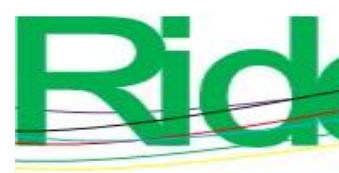

Revista Iberoamericana para la
Investigación y el Desarrollo Educativo
ISSN $2007-7467$

Morales, V. G. (2013). Desarrollo de competencias digitales docentes en la educación básica. Apertura, $5(1)$.

Recuperado de http://www.udgvirtual.udg.mx/apertura/index.php/apertura/article/view/367/307\#resu.

Muñoz, M. (2011). El uso de las TIC's en EMS. Visión de un grupo de profesores-estudiantes. Revista Mexicana de Bachillerato a Distancia, 5(3), 84-93.

Otzen, T. y Manterola, C. (2017). Técnicas de muestreo sobre una población a estudio. International Journal of Morphology, 35(1), 227-232.

Razo, A. E. y Cabrero, I. (2015). Uso y organización del tiempo en aulas de educación media superior. Ciudad de México, México: Secretaría de Educación Pública.

Rodríguez, J. L., Martínez, N. y Lozada, J. M. (2009). Las TIC como recursos para un aprendizaje constructivista. Revista de Artes y Humanidades UNICA, 10(2), 118-132.

Santiago, G., Caballero, R., Gómez, D. y Domínguez, A. (2013). El uso didáctico de las TIC en escuelas de educación básica en México. Revista Latinoamericana de Estudios Educativos (México), 43(3), 99-131.

Takabayashi, T. (2017). Expectations of learning media for self-directed learning: Learners' perceptions of media choice. International Journal for Educational Media and Technology, 11(1), 59-67.

Tamayo, G. (2001). Diseños muestrales en la investigación. Semestre Económico, 4(7), 1-14.

Terashima, K., Nakagawa, H., Kobayashi, Y. and Murai, M. (2019). Technology integration changes over three years: teacher technology acceptance in a one-to-one tablet PC integration across cities. International Journal for Educational Media and Technology, 13(1), 17-26.

Zenteno, A. y Mortera, F. (2011). Integración y apropiación de las TIC en los profesores y alumnos de educación media superior. Apertura, 3(1). 